Check for updates

Cite this: RSC Adv., 2017, 7, 35252

Received 4th May 2017

Accepted 10th July 2017

DOI: $10.1039 / \mathrm{c} 7 \mathrm{ra05028c}$

rsc.li/rsc-advances

\section{Specific microwave effect on Sn- and Ti-MFI zeolite synthesis $\uparrow$}

\author{
Zhen Sun, Ting Li, Gang Li, Yahong Zhang (D) and Yi Tang (DD) \\ A specific microwave effect on the $\mathrm{Sn}$ - and Ti-MFI zeolite synthesis is exactly demonstrated by separating it \\ from thermal effects through using Pyrex and $\mathrm{SiC}$ reaction vessels. Different from the reported conventional \\ thermal effect on the organic reactions, this finding is of great significance to further investigate the \\ microwave effect.
}

Since microwave energy was used to heat organic reactions in $1986,{ }^{1-3}$ the advantages of this efficient heating tool have been exploited in the field of organic synthesis, medical chemistry, polymer chemistry, nanostructured material and microporous material synthesis..$^{4-8}$ In most cases, microwave heating has been shown to dramatically reduce processing time, increase product yield, and enhance product purity or materials properties compared with the conventional heating mode. The observed improvement of chemical reaction or materials synthesis have often led to speculations about the so-called "specific" or "nonthermal" microwave effect that results from a proposed direct interaction between the electromagnetic field and specific molecules in the reaction medium. However, so far, more and more experiments assigned this effect to inaccurate temperature measurement, inadequate stirring, imprecise comparisons of microwave irradiation and conventional heating. ${ }^{9-11}$ After more than a decade of intense research, Kappe et al. designed a clever experiment to separate specific and nonthermal effects from pure thermal effect. They used a strongly microwave-absorbing material silicon carbide (SiC) reaction vessel in which any effects of the electromagnetic field on the reaction mixture can be eliminated. ${ }^{12-14}$ The SiC vessel can also be heated simultaneously, together with a single mode microwave reactor equipped with an external infrared (IR) and an internal fiber-optic (FO) probes for accurate temperature monitoring. ${ }^{15}$ Applied to many organic reactions, however, they proposed that only bulk temperature effects were responsible for the rate enhancement in the field of organic reactions. ${ }^{16,17}$

Department of Chemistry, Shanghai Key Laboratory of Molecular Catalysis and Innovative Materials, Laboratory of Advanced Materials, Collaborative Innovation Centre of Chemistry for Energy Materials, Fudan University, Shanghai 200433, P. R. China. E-mail: zhangyh@fudan.edu.cn

$\dagger$ Electronic supplementary information (ESI) available: Experimental section, ramping time of reaction system in Pyrex and SiC vessels. Temperature and power profiles for Sn-MFI system, a serious of parameters for the reaction solution in the Pyrex and SiC vessels, more detailed XRD, DR UV-Vis, Argon sorption isotherm, NLDFT pore size distribution and nonaqueous potentiometric titration curves with $n$-butylamine of Sn- and Ti-MFI zeolites obtained in Pyrex and SiC vessels. See DOI: 10.1039/c7ra05028c
However, the finding of specific/nonthermal microwave effect is still highly desired because of the fact that microwave heating is carried out by direct interaction between the electromagnetic field and polar molecules, and different dipolar molecules possess different abilities of converting electromagnetic energy into heat. ${ }^{18-20}$

The isomorphous substitution of heteroatoms for $\mathrm{Si}$ in the zeolite framework is an important process because it allows the fine tuning of their properties. For the microwave assistant synthesis of heteroatom zeolites, Cundy et al. did pioneering works including TS-1 and TS-2 synthesis. ${ }^{21}$ Moreover, microwave irradiation has been reported to be responsible for the synthesis of heteroatom zeolite with special morphologies, ${ }^{22}$ which is assigned to the dipole moment strength of the surface $\mathrm{M}-\mathrm{O}$ bonds. However, indicated by Kappe, ${ }^{10,11}$ inaccurate comparison often led to misinterpretation of results. In this work, we rigorously investigate the synthetic processes of Snand Ti-MFI zeolites as well as resultant texture properties monitored by using $\mathrm{SiC}$ vessel and accurate temperature measurement. By simply switching from a standard microwave transparent borosilicate glass (Pyrex) to a strongly microwave absorbing SiC vial, effects of the electromagnetic field on the reaction mixture could be minimized, therefore allowing to distinguish the specific microwave effect from the thermal one. Under this precise comparison, the different crystallization rates, existences of heteroatom in the framework, as well as catalytic performances of the resulting products obtained in two vessels definitely demonstrated the specific microwave effect on the heteroatom zeolite preparation. We also assign this effect to the selective activation of microwave on polar $\mathrm{Sn}-\mathrm{O}$ and $\mathrm{Ti}-\mathrm{O}$ bonds.

To avoid the interference of thermal and other factors, we adopted the same experimental conditions as Kappe, that is, microwave transparent Pyrex vessel and strong microwaveabsorbing SiC vessel as well as a dedicated single-mode microwave reactor equipped with the external IR and the internal FO probes under magnetic stirring. Before comparing, heating rate of Pyrex and SiC vessels should also be guaranteed basically the same. According to the synthetic process of 
heteroatom MFI zeolite (Experimental section, ESI $\dagger$ ), the temperature of $80{ }^{\circ} \mathrm{C}$ and $170{ }^{\circ} \mathrm{C}$ are defined as low temperature zone and high temperature zone, respectively. As shown in Fig. 1 and S1 (ESI $\dagger$ ), the rise rate of internal FO temperature is faster than external IR temperature in the Pyrex vessel system, whereas the former is slower than the latter in the SiC vessel. As illustrated in Fig. 1c and f, the reaction mixture directly absorbs the microwave in Pyrex vessel, and then the heat generates and conducts to the outside wall. Therefore, it will take some time for the vessel to be warmed "from the inside". On the contrary, in the SiC vessel, SiC converts the completely absorbed microwave into heat and then conduct inside, leading to the delayed response of FO probe. However, the ramping time of reaction system in Pyrex and SiC vessels are basically the same whether in low or high temperature zone (Table S1, ESI $\dagger$ ). Furthermore, the specific experiment parameters are depicted in Fig. S2 $(\mathrm{ESI} \dagger)$, which shows the stable reaction process. All above confirm that the investigation can be operated in parallel in the dedicated microwave reactor equipped with FO probe and the two vessels.

The synthesis processes of Sn- and Ti-MFI zeolites in two vessels are monitored by an approximate in situ dynamic light scattering (DLS) measurement. The effective diameters of asprepared zeolites at different crystallization time are obtained from number-weighted particle size distribution. Variations of particle diameter as a function of crystallization time of Sn- and Ti-MFI are shown in Fig. 2 (termed as growth curves). Clearly, there is a sudden pronounced rise in particle diameter observed in each curve. We regard the time before the inflection point as nucleation period, and that after the inflection point as crystal growth period. In detail, Sn-MFI zeolites in two vessels experience a long nucleation period and a slow growth process to achieve their stable sizes (Fig. 2a). However, the nucleation and growth of Sn-MFI in SiC vessel are slower than those in Pyrex vessel. Moreover, the final stable size of the product in Pyrex is
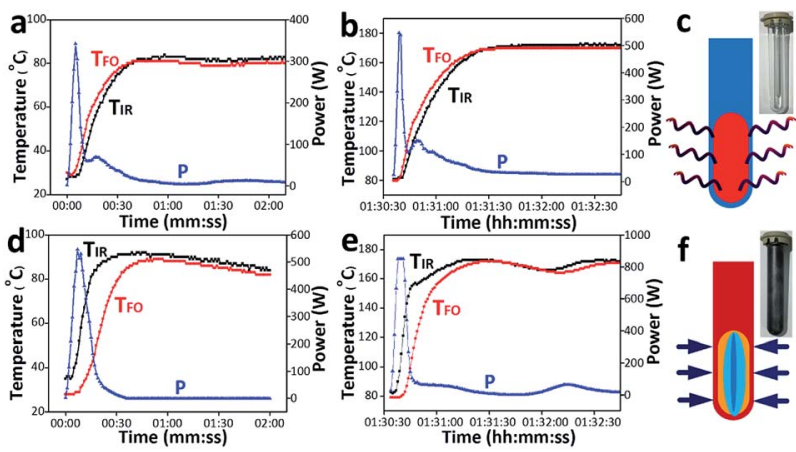

Fig. 1 Temperature $\left(T_{\mathrm{IR}}, T_{\mathrm{FO}}\right)$ and power $(P)$ profiles in the ramping phase recorded for the reaction system for Ti-MFI in the Pyrex ( $(\mathrm{a})$ from room temperature to $80^{\circ} \mathrm{C}$, (b) from $80^{\circ} \mathrm{C}$ to $170{ }^{\circ} \mathrm{C}$ ) and $\mathrm{SiC}$ vessel ((d) from room temperature to $80^{\circ} \mathrm{C}$, (e) from $80^{\circ} \mathrm{C}$ to $170{ }^{\circ} \mathrm{C}$ ), respectively, heated with microwave under sealed-vessel conditions in the Monowave 300 reactor. Schematic diagram of heat transfer mode in Pyrex (c) and SiC (f) vessels (the vial photos show in the insets, and the red colour and blue colour denote high and low temperature, respectively).
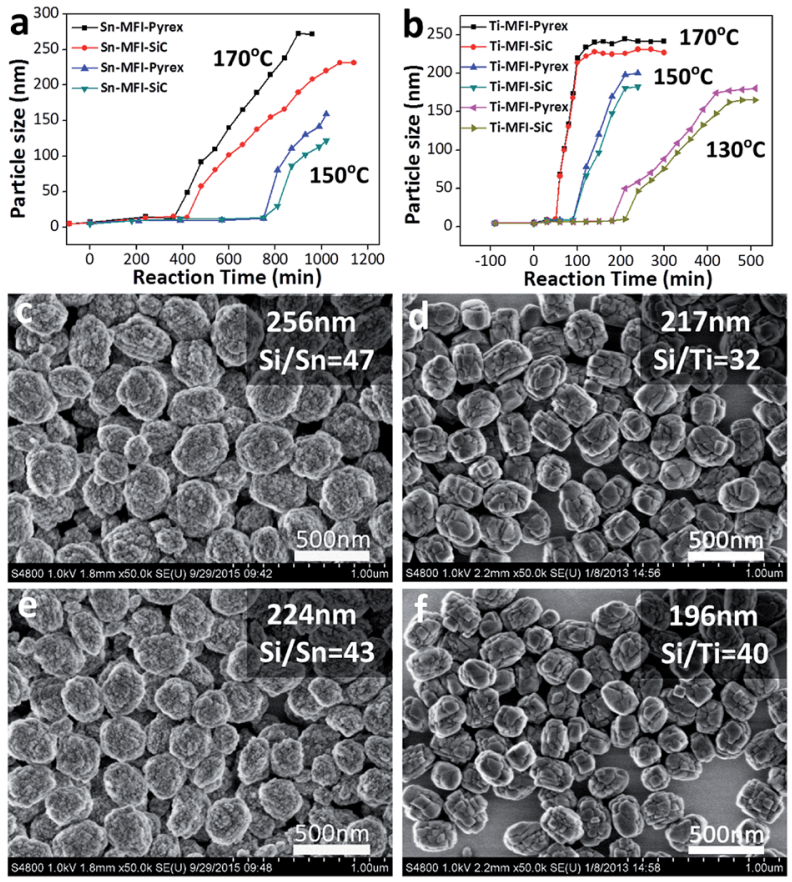

Fig. 2 Variation of particle diameter as a function of crystallization time of Sn-MFI (a) and Ti-MFI (b) zeolites prepared under microwave irradiation in Pyrex and $\mathrm{SiC}$ systems (the time interval before 0 at the $x$ axis represents that of the microwave pre-treatment at $80{ }^{\circ} \mathrm{C}$ for $90 \mathrm{~min}$, whereas that after 0 represents the microwave treatment time at different temperatures). FESEM images of Sn- and Ti-MFI synthesized in Pyrex (c, d) and $\operatorname{SiC~(e,f)~vessels,~respectively~(high~temperature~}$ zone: $170{ }^{\circ} \mathrm{C}$ ). The average particle size and Si/M ratios of corresponding products are shown in the insets, which are obtained based on their FESEM images and energy dispersive X-ray (EDX) data, respectively.

much larger than that in SiC. Although Ti-MFI zeolites, by contrast, display a comparatively quick nucleation period and growth rate to reach their stable sizes, the average size of Ti-MFI obtained in Pyrex is also bigger than the one in SiC finally (Fig. 2b). Moreover, this difference of crystallization rate and final particle size between reaction vessels exists at different crystallization temperatures (Fig. 2a and b). Furthermore, the size differences between two vessels can be further confirmed by their average solid sizes calculated by a size-calculated software on the basis of field emission scanning electron microscope (FESEM) images (Fig. 2c-f), although they are slightly smaller than those in the solution. These results imply that the direct interaction between the electromagnetic field and reaction medium, except for its thermal effect, possesses positive influence on the preparation process of Sn- and Ti-MFI zeolites.

However, it is worthy to note that when no any heteroatom (Sn or Ti) species is added during MFI zeolite synthesis, nearly no difference between two synthesis systems can be observed even if the crystallization temperature is decreased to $130{ }^{\circ} \mathrm{C}$ (Fig. 3). Therefore, we deduce that this microwave effect is derived from the interaction between microwave field and heteroatom species. Moreover, taking microwave heating principle and previous reports into consideration, ${ }^{17,24}$ we assign this effect to the microwave selective heating for $\mathrm{Sn}-\mathrm{O}$ and $\mathrm{Ti}-\mathrm{O}$ 


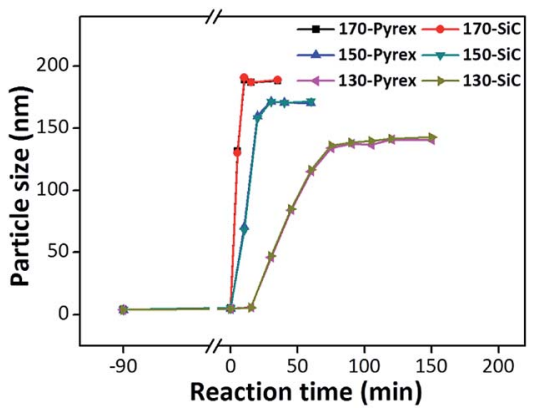

Fig. 3 Crystal growth curves of silicalite-1 in Pyrex and SiC vessels synthesized at different temperatures.

bonds. In the microwave field, different dipolar molecules or bonds possess different wave-absorption abilities. The dipole moment of $\mathrm{M}-\mathrm{O}$ bond can be denoted by the electronegativity differences $(\Delta \chi)$ between $\mathrm{M}$ and $\mathrm{O}$ atoms. Therefore, the polar $\mathrm{Sn}-\mathrm{O}(\Delta \chi=1.78)$ and $\mathrm{Ti}-\mathrm{O}(\Delta \chi=2.18)$ bonds with bigger dipole moment possess stronger wave-absorption abilities than $\mathrm{Si}-\mathrm{O}$ $(\Delta \chi=1.76)$ bond in the microwave field, causing the localized high temperature around $\mathrm{Sn}-\mathrm{O}$ and $\mathrm{Ti}-\mathrm{O}$ bonds. Consequently, microwave field can selectively heat and activate the $\mathrm{Sn}-\mathrm{O}$ and Ti-O bonds in the synthetic gel, solidly promoting their substitution and influencing their state in the zeolite framework. When no heteroatom is added, this specific microwave effect disappears accordingly. These results mean that different from thermal effect, the specific microwave effect is not a general rule, and it only selectively exists in some suitable system where the influence of electromagnetic field can be accumulated.

Besides, as-prepared Sn-MFI and Ti-MFI zeolites obtained under two reaction vessels are further characterized to study the difference between two heating modes. Herein, M-MFI prepared in Pyrex and SiC vessels are denoted as M-MFI-Pyrex and M-MFISiC, respectively. X-ray diffraction (XRD) patterns (Fig. S3, ESI $\dagger$ ) confirm their typical MFI framework without impurity phase. However, compared with the Sn-MFI-Pyrex sample, Sn-MFI-SiC displays a lower crystallinity of $94 \%$. Similarly, compared with the Ti-MFI-Pyrex, Ti-MFI-SiC shows a lower crystallinity of $96 \%$. The diffuse reflectance (DR) UV-Vis spectra of them show the bands within the range of 203-211 nm (Fig. S4, ESI†), implying the existence of tetrahedral Sn or Ti species in the MFI framework. ${ }^{23-25}$ These results collectively indicate that the Sn and Ti species can be substituted into the MFI framework in both Pyrex and $\mathrm{SiC}$ vessels. However, the X-ray photoelectron spectroscopy (XPS) results of samples obtained in two reaction vessels, representing existence and surface local state of heteroatom in the zeolite framework, exhibit a difference (Fig. 4). The binding energy (BE) of Sn-MFI-Pyrex in the Sn 3d region are 496.2 and $487.6 \mathrm{eV}$, which are ascribed to $3 \mathrm{~d}_{3 / 2}$ and $3 \mathrm{~d}_{5 / 2}$ photoelectrons of tetrahedrally coordinated framework Sn species. ${ }^{26,27}$ However, the corresponding BEs (496.0 and $487.2 \mathrm{eV}$ ) of Sn-MFI-SiC are lower than those of Sn-MFI-Pyrex, demonstrating stronger covalent bond of Sn-O-Si in Sn-MFI-Pyrex zeolite. ${ }^{27}$ Similarly,
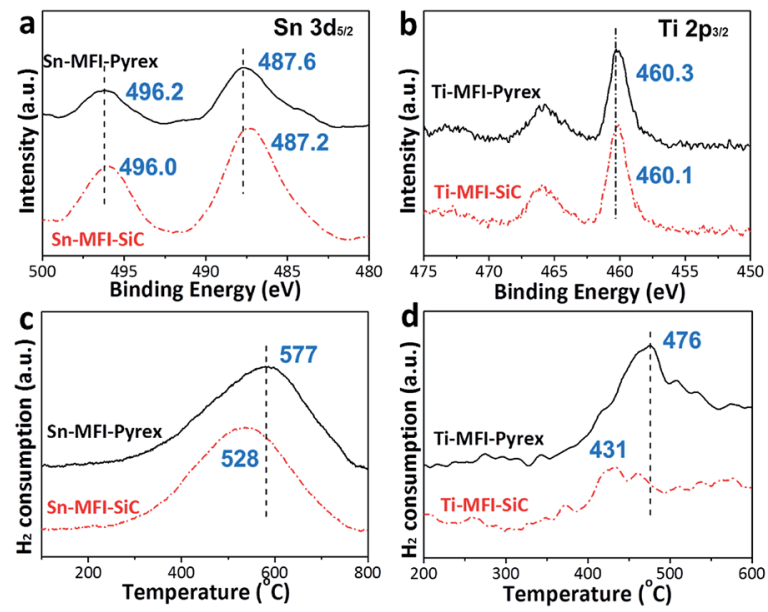

Fig. 4 XPS spectra (a, b) and $\mathrm{H}_{2}$-TPR spectra (c, d) of Sn-MFI (a, c) and Ti-MFI (b, d) zeolites prepared in Pyrex and SiC systems.

the $\mathrm{BE}$ of $\mathrm{Ti} 2 \mathrm{p}_{3 / 2}$ in Ti-MFI-Pyrex $(460.3 \mathrm{eV})$ is also higher than that of Ti-MFI-SiC (460.1 eV).

Furthermore, different reduction temperatures between SnMFI-Pyrex and Sn-MFI-SiC, as well as Ti-MFI-Pyrex and TiMFI-SiC are clearly observed in temperature-programmed reduction by hydrogen $\left(\mathrm{H}_{2}\right.$-TPR). The results of TPR data in Fig. $4 \mathrm{c}$ and $\mathrm{d}$ indicate that $\mathrm{Sn}$ - and Ti-MFI-Pyrex zeolites require higher temperature than those prepared in SiC. Therefore, taking their comparable heteroatom amounts in M-MFI zeolites prepared under the two reaction systems into consideration (Fig. 2c-f), the offsets of the reduction peaks indicate their more stable heteroatom framework states in the zeolites. ${ }^{28}$ These further illustrate that electromagnetic field displays a direct influence on the heteroatom zeolite synthesis, not only the crystallization rate and particle size but also product property.

Additionally, the great difference between the resultant $\mathbf{M}$ MFI-Pyrex and M-MFI-SiC zeolites on the catalytic performance further demonstrate the importance of this specific microwave effect. It is well known that Sn-containing zeolite appears to be active in the Meerwein-Ponndorf-Verley (MPV) reduction. ${ }^{29,30}$ Herein, the MPV reduction of furfural (FUR) in isopropanol is selected as probe reaction to evaluate the catalytic activity of Sn-MFI zeolites prepared in Pyrex and SiC vessels (Fig. 5a). As shown in Fig. 5a, the conversion of FUR catalysed by Sn-MFI-Pyrex is clearly higher than that catalysed by Sn-MFISiC. In detail, more than $64 \%$ conversion of FUR can be reached on Sn-MFI-Pyrex catalyst within $5 \mathrm{~h}$. In this case, turn over frequency (TOF) number is as high as $9.8 \mathrm{~h}^{-1}$ by considering $\mathrm{Sn}$ as the only active sites. However, only $53 \%$ of conversion and TOF of $6.8 \mathrm{~h}^{-1}$ are achieved on Sn-MFI-SiC within the same time, moreover, during the experiments, it is found that the further etherification of reduced furfuryl alcohol (FOL) occurs with the MPV reduction of FUR, and selectivity of i-propyl-furfuryl ether (PFE) increases with reaction time whereas that of FOL decreases (Fig. 5a). The higher catalytic activity of Sn-MFI-Pyrex can be assigned to larger micropore area (Fig. S5a, b and Table S2, ESI $\dagger$ ), slightly stronger Lewis 


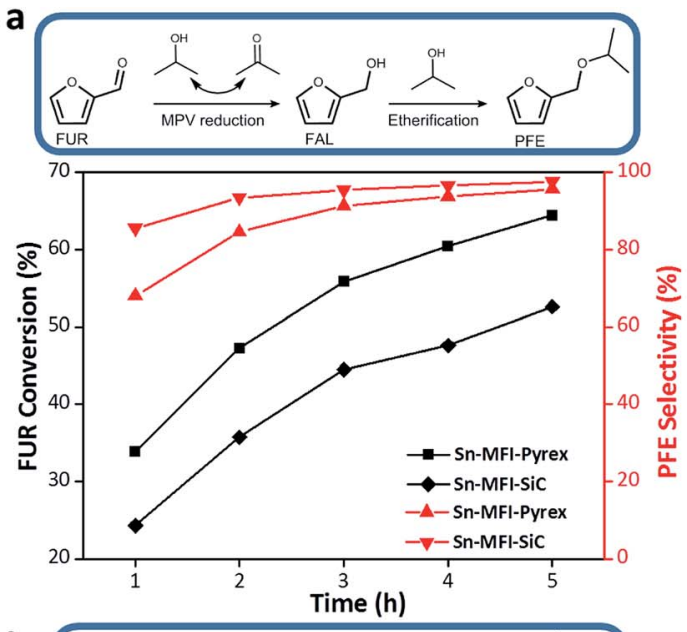

b
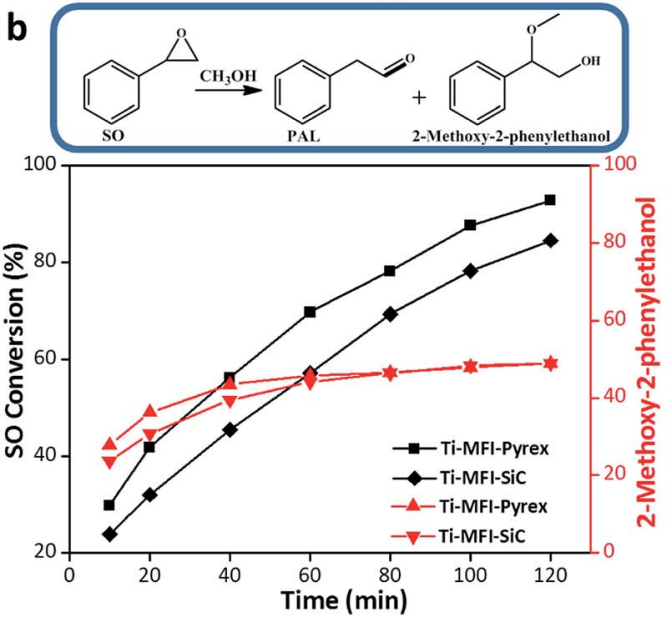

Fig. 5 (a) Conversion of furfural and selectivity of i-propyl-furfuryl ether in the reactions with Sn-MFI-Pyrex and Sn-MFI-SiC catalysts. Reaction conditions: $1 \mathrm{mmol}$ furfural, $100 \mathrm{mmol}$ isopropanol, $100 \mathrm{mg}$ catalyst, at $100^{\circ} \mathrm{C}$; (b) conversion of styrene oxide and selectivity of 2 methoxy-2-phenylethanol in the reactions with Ti-MFI-Pyrex and TiMFI-SiC catalysts. Reaction conditions: $1 \mathrm{mmol}$ styrene oxide, $60 \mathrm{mmol}$ methanol, $20 \mathrm{mg}$ catalyst, at $80^{\circ} \mathrm{C}$.

acidity (Fig. S6a and Table S2, ESI $\dagger$ ) and its effective heteroatom sites (Fig. 4).

Besides, ring-opening of epoxide reaction is employed to evaluate the catalytic activity of Ti-MFI zeolites prepared by two vessels. $^{31}$ The conversion of styrene oxide (SO) catalysed by TiMFI-Pyrex is clearly higher than that catalysed by Ti-MFI-SiC (Fig. 5b). In detail, $92 \%$ and $84 \%$ of SO conversions were reached on Ti-MFI-Pyrex and Ti-MFI-SiC catalysts within $2 \mathrm{~h}$, respectively. Moreover, Ti-MFI-Pyrex also possesses a higher TOF number $\left(202.7 \mathrm{~h}^{-1}\right)$ than Ti-MFI-SiC $\left(165.8 \mathrm{~h}^{-1}\right)$. The main products are phenyl acetaldehyde (PAL) and 2-methoxy-2phenylethanol, and the selectivity of 2-methoxy-2-phenylethanol increases with the prolonging reaction time (Fig. 5b). Because there is little difference of textural properties between Ti-MFIPyrex and Ti-MFI-SiC (Fig. S5c, d and Table S2, ESI $\dagger$ ), the higher catalytic activity of Ti-MFI-Pyrex can be assigned to its more stable and effective heteroatom framework sites (Fig. 4), and higher Lewis acidity (Fig. S6b and Table S2, ESI $\dagger$ ) than
Ti-MFI-SiC. These catalytic results further demonstrate the existence of the specific microwave effect on the heteroatom zeolite synthesis as well as its important and positive influence on heteroatom sites in the zeolite framework.

\section{Conclusions}

In summary, transparent Pyrex and strongly microwaveabsorbed SiC vessels are utilized to separate specific/ nonthermal microwave effect from the thermal one during the synthesis of Sn- and Ti-MFI zeolites. After accurately comparing the differences of Sn- and Ti-MFI prepared in two systems, it concluded that there is specific microwave effect during the synthesis of Sn- and Ti-MFI zeolites, produced by selective activation on the $\mathrm{M}-\mathrm{O}$ bonds, which not only definitely exists but also has a positive effect on the substitution of heteroatom in the zeolite framework and their catalytic performances, which is very significant for further investigating some microwave effects in the future.

\section{Conflicts of interest}

There are no conflicts of interest to declare.

\section{Acknowledgements}

This work was supported by the 973 Program (2013CB934101) and NSFC (21171041, 21473037, U1463206).

\section{Notes and references}

1 S. Komarneni and R. Roy, Mater. Lett., 1985, 3, 165-167.

2 M. R. Rosana, Y. C. Tao, A. E. Stiegman and G. B. Dudley, Chem. Sci., 2012, 3, 1240-1244.

3 M. Tsuji, M. Hashimoto, Y. Nishizawa, M. Kubokawa and T. Tsuji, Chem.-Eur. J., 2005, 11, 440-452.

4 A. de la Hoz, A. Diaz-Ortiz and A. Moreno, Chem. Soc. Rev., 2005, 34, 164-178.

5 C. O. Kappe and D. Dallinger, Nat. Rev. Drug Discovery, 2006, 5, 51-63.

6 K. Kempe, C. R. Becer and U. S. Schubert, Macromolecules, 2011, 44, 5825-5842.

7 I. Bilecka and M. Niederberger, Nanoscale, 2010, 2, 13581374.

8 A. M. Schwenke, S. Hoeppener and U. S. Schubert, Adv. Mater., 2015, 27, 4113-4141.

9 N. Kuhnert, Angew. Chem., Int. Ed., 2002, 41, 1863-1866.

10 C. O. Kappe, B. Pieber and D. Dallinger, Angew. Chem., Int. Ed., 2013, 52, 1088-1094.

11 M. Baghbanzadeh, S. D. Skapin, Z. C. Orel and C. O. Kappe, Chem.-Eur. J., 2012, 18, 5724-5731.

12 M. A. Herrero, J. M. Kremsner and C. O. Kappe, J. Org. Chem., 2008, 73, 36-47.

13 T. Razzaq, J. M. Kremsner and C. O. Kappe, J. Org. Chem., 2008, 73, 6321-6329. 
14 J. Robinson, S. Kingman, D. Irvine, P. Licence, A. Smith, G. Dimitrakis, D. Obermayer and C. O. Kappe, Phys. Chem. Chem. Phys., 2010, 12, 10793-10800.

15 C. O. Kappe, Chem. Soc. Rev., 2013, 42, 4977-4990.

16 C. R. Strauss and D. W. Rooney, Green Chem., 2010, 12, 13401344.

17 C. O. Kappe, Chem. Soc. Rev., 2008, 37, 1127-1139.

18 M. Baghbanzadeh, L. Carbone, P. D. Cozzoli and C. O. Kappe, Angew. Chem., Int. Ed., 2011, 50, 11312-11359. 19 J. Klinowski, F. A. Paz, P. Silva and J. Rocha, Dalton Trans., 2011, 40, 321-330.

20 D. M. P. Mingos and D. R. Baghurst, Chem. Soc. Rev., 1991, 20, 1-47.

21 C. S. Cundy and J. O. Forrest, Microporous Mesoporous Mater., 2004, 72, 67-80.

22 Y. K. Hwang, J.-S. Chang, S.-E. Park, D. S. Kim, Y.-U. Kwon, S. H. Jhung, J.-S. Hwang and M. S. Park, Angew. Chem., Int. Ed., 2005, 44, 557-560.

23 N. K. Mal and A. V. Ramaswamy, J. Mol. Catal. A: Chem., 1996, 105, 149-158.
24 W. B. Fan, R. G. Duan, T. Yokoi, P. Wu, Y. Kubota and T. Tatsumi, J. Am. Chem. Soc., 2008, 130, 10150-10164.

25 P. Li, G. Q. Liu, H. H. Wu, Y. M. Liu, J. G. Jiang and P. Wu, J. Phys. Chem. C, 2011, 115, 3663-3670.

26 B. Tang, W. L. Dai, G. J. Wu, N. J. Guan, L. D. Li and M. Hunger, ACS Catal., 2014, 4, 2801-2810.

27 J. Dijkmans, M. Dusselier, D. Gabriëls, K. Houthoofd, P. C. M. M. Magusin, S. G. Huang, Y. Pontikes, M. Trekels, A. Vantomme, L. Giebeler, S. Oswald and B. F. Sels, ACS Catal., 2015, 5, 928-940.

28 J. Dijkmans, M. Dusselier, W. Janssens, M. Trekels, A. Vantomme, E. Breynaert, C. Kirschhock and B. F. Sels, ACS Catal., 2016, 6, 31-46.

29 J. Iglesias, J. A. Melero, G. Morales, J. Moreno, Y. Segura, M. Paniagua, A. Cambra and B. Hernández, Catalysts, 2015, 5, 1911-1927.

30 D. Padovan, C. Parsons, M. S. Grasina and C. Hammond, Green Chem., 2016, 18, 5041-5049.

31 R. Kore, R. Srivastava and B. Satpati, ACS Catal., 2013, 3, 2891-2904. 